\title{
新型吡啶氧基苯胺基丙酸/乙酸乙酯的合成以及除草活性研究
}

\author{
徐志红 $a$ 张 涛 $b$ 王少康 $b$ 李俊凯*, $a$ \\ $\left({ }^{a}\right.$ 长江大学 主要粮食作物产业化湖北省协同创新中心 荆州 434205) \\ $\left({ }^{b}\right.$ 长江大学农学院 荆州 434205)
}

\begin{abstract}
摘要 芳氧苯氧基丙酸酯是一类以单子叶植物叶绿体内乙酰辅酶 $\mathrm{A}$ 羧化酶为作用靶标的重要除草剂. 合成了 23 个全新 的吡啶氧基苯胺基丙酸/乙酸乙酯类化合物，并通过核磁共振氢谱、高分辨质谱和 $\mathrm{X}$ 单晶衍射对新化合物结构进行了确 证. 测定了新化合物对稗草和油菜的室内生物活性, 结果表明, 新化合物对稗草具有较好的抑制作用, 其中 2-[4-(5$\mathrm{NO}_{2}$-2-吡啶氧基)苯胺基]丙酸乙酯(B5)活性最高, 对稗草的除草活性与对照药剂氟吡甲禾灵相当, 可以作为研究新除草 剂的先导化合物.
\end{abstract}

关键词 吡啶氧基苯胺基丙酸/乙酸乙酯; 合成; 除草活性; 乙酰辅酶 $\mathrm{A}$ 羧化酶

\section{Synthesis and Herbicidal Activities of Novel Ethyl 2-(4-(Pyridin-2-yl-oxy)phenyl-amino)propanoates/acetates}

\author{
Xu, Zhihong ${ }^{a} \quad{\text { Zhang, } \text { Tao }^{b} \quad \text { Wang, Shaokang }}^{b} \quad$ Li, Junkai ${ }^{*, a}$ \\ ( ${ }^{a}$ Hubei Collaborative Innovation Center for Grain Industry, Yangtze University, Jingzhou 434205) \\ ( ${ }^{b}$ Agricutural College, Yangtze University, Jingzhou 434205)
}

\begin{abstract}
Aryloxyphenoxypropionates (APPs) are a class of herbicides targeting on acetyl-coenzyme A carboxylase (ACCase) in monocot chloroplast. The article presents synthesis of twenty three novel ethyl 2-(4-(pyridin-2-yl-oxy)phenylamino)propanoates/acetates, and their structures were characterized by ${ }^{1} \mathrm{H}$ NMR, HRMS and X-ray single-crystal diffraction. The herbicidal activities on barnyard grass and rape of the novel compounds were evaluated. Ethyl 2-(4-(5-nitropyridin-2-yloxy)phenylamino)propanoate (B5) showed almost the same level of herbicidal activity on barnyard grass as commercial herbicide fluazifop-methyl which could become a potential lead compound on weeds.

Keywords ethyl 2-(4-(pyridin-2-yl-oxy)phenylamino)propanoate/acetates; synthesis; herbicidal activity; acetyl-CoA carboxylase
\end{abstract}

芳氧苯氧基丙酸酯类化合物是一类广泛使用的苗 后除草剂, 主要防除阔叶作物田的一年生和多年生杂 草. 这类化合物的除草活性在 20 世纪 70 年代就发现了, 从那时起就有大量的研究用以阐明作用机理和作用靶 标. 人们认为其作用靶标为植物的乙酰辅酶 $\mathrm{A}$ 羧化酶 (ACCase, E.C. 6.4.1.2) ${ }^{[1 \sim 4]}$, 通过抑制禾本科植物体内脂 类的合成而防除禾本科杂草. 至今, 已经有超过 20 种芳 氧苯氧基丙酸酯类除草剂商品化, 包括炔草酯 ${ }^{[5]}$ 、噁唑 禾草灵 ${ }^{[6]}$ 、吡氟禾草灵 ${ }^{[7,8]}$ 、氟吡甲禾灵 ${ }^{[5]}$ 、喹禾灵 ${ }^{[9]}$ 、 氧氟草酯 ${ }^{[10]}$ 、噁唑酰草胺 ${ }^{[11]}$ 等, 并由世界主要的农药公 司推向市场, 如图 1 所示. 这些除草剂在大豆、小麦、
水稻等作物田使用, 防除禾本科杂草 ${ }^{[12]}$.

1997 年报道了一系列 $N$-甲基取代的芳胺苯氧基丙 酸酯类化合物, 与芳氧苯氧基丙酸酯类化合物相比, 具 有更高的除草活性. 用 $N$-甲基作为一个新生物等排体, 当芳基为 6 -氯喹啉和 7 -氯苯 $[1,2,4]$ 三嗪时表现出特别高 的除草活性 ${ }^{[13]}$. 刘钊杰等 ${ }^{[14]}$ 对含有芳氧苯氧基丙酸酯 基团的新型吡唑并嘧啶-4-酮衍生物进行了除草活性试 验，发现这类化合物具有除草活性，并可作为一种潜在 的除草剂进行深入研究. 张爱东等 ${ }^{[15]}$ 报道了多个含有 2嘧啶和 4-嘧啶基团的芳氧苯氧基丙酸酯类化合物, 并对 其除草活性进行了测定, 发现对稗草茎生长的抑制率高

\footnotetext{
* Corresponding author. E-mail: junkaili@sina.com

Received August 23, 2016; revised September 6, 2016; published online October 9, 2016.

Project supported by the National Natural Science Foundation of China and the Open Fund for Hubei Collaborative Innovation Center for Grain Industry.

国家自然科学基金(No. 31301976)和主要粮食作物产业化湖北省协同创新中心开放基金资助项目.
} 


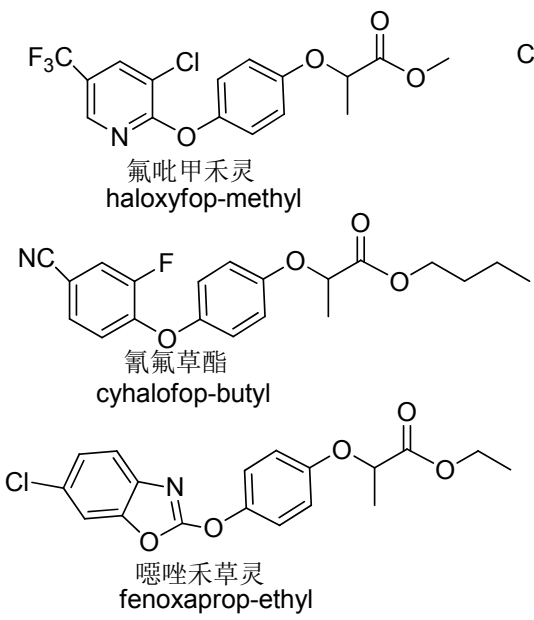<smiles>CC(C)=NOCCOC(=O)C(C)Oc1ccc(Oc2cnc3cc(Cl)ccc3n2)cc1</smiles><smiles>CC(Oc1ccc(Oc2cnc3cc(Cl)ccc3n2)cc1)C(=O)OCC1CCCO1</smiles><smiles>CC(Oc1ccc(Oc2nc3ccc(Cl)cc3o2)cc1)C(=O)N(C)c1ccccc1F</smiles>

图 1 芳氧苯氧基丙酸酯类除草剂的结构

Figure 1 Structures of APPs herbicides

于氰氟草酯.

根据上述文献的启示, 特别是氟吡甲禾灵结构与高 除草活性的关系, 选择芳氧苯氧丙酸酯类化合物中的氟 吡甲禾灵为先导化合物, 根据生物等排性原理, 引入了 芳氧苯胺基的结构, 设计含有吡啶氧基苯胺基丙酸/乙 酸乙酯类结构的一系列化合物. 这种结构可能具有芳氧 苯氧丙酸酯类化合物高效、速效、杀草谱广、不易产生 抗性等优点. 同时, 该化合物可能被降解并释放出二苯 醚的结构, 进一步抑制杂草的生长. 这类化合物的研究 有较大理论意义和应用前景.

\section{1 结果与讨论}

\section{1 化合物合成}

当中间体 $\mathbf{A}$ 进攻 2-氯丙酸乙酯时，只能得到单取代 的胺基化合物 $\mathbf{B} 1 \sim \mathbf{B} 18$, 这是由于 2 -氯丙酸乙酯具有较 大的空间位阻. 而当中间体 $\mathbf{A}$ 进攻 2-氯乙酸乙酯时，可 以分别得到单取代的化合物 $\mathbf{C 1} \sim \mathbf{C 3}$ 和双取代的化合物 $\mathbf{D}$ 与 $\mathbf{F}$. 由于吡啶环上 3 位的氟比 2 位的氯更容易发生 反应，所以得到的化合物 $\mathbf{F}$ 是 3 位取代的而不是 2 位. 化合物 $\mathbf{F}$ 的晶体结构(图 2)显示取代的正是吡啶环上的 3 位，而且在氮上进行的是双取代.

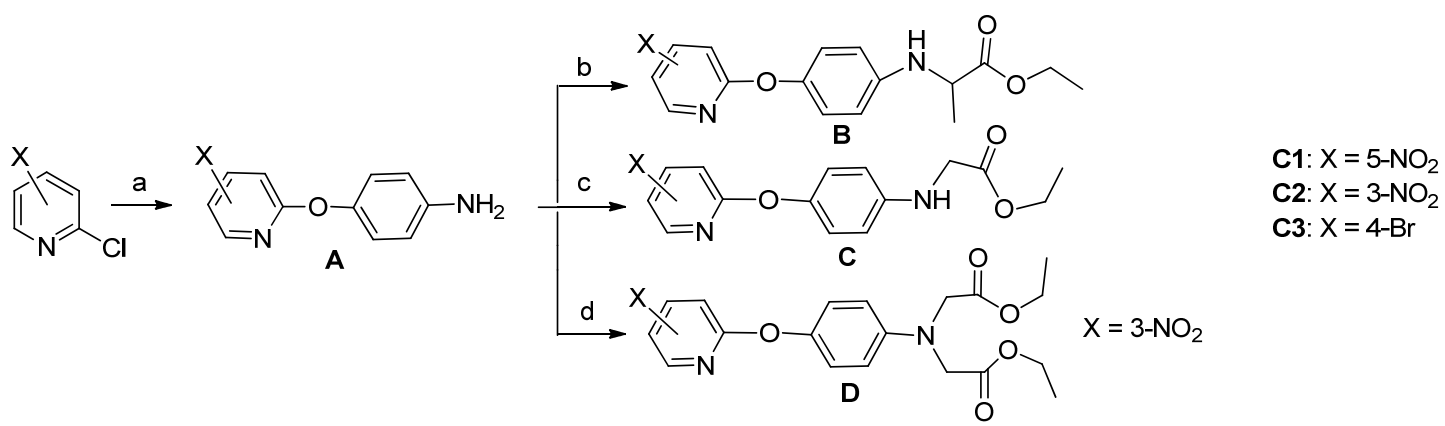

Reagents and conditions: (a) 4-aminophenol, sodium tert-butoxide; (b) KI, ethyl 2-chloro-propanoate (1 equiv.); (c) KI, ethyl 2-chloroacetate (1 equiv.); (d) $\mathrm{KI}$, ethyl 2-chloroacetate (3 equiv.).

图式 1 化合物 B1 B18, C1 C $~$ 和 D 的合成路线

Scheme 1 Synthetic route for compounds $B 1 \sim B 18, C 1 \sim C 3$ and $D$
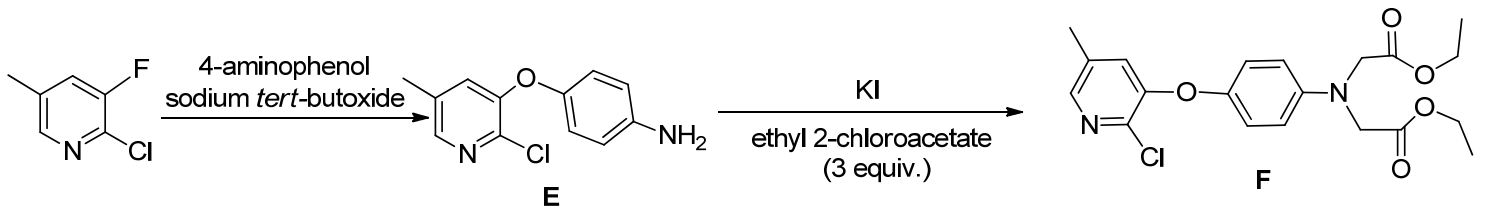

图式 2 化合物 $\mathbf{F}$ 的合成路线.

Scheme 2 Synthetic route for compound $\mathbf{F}$ 


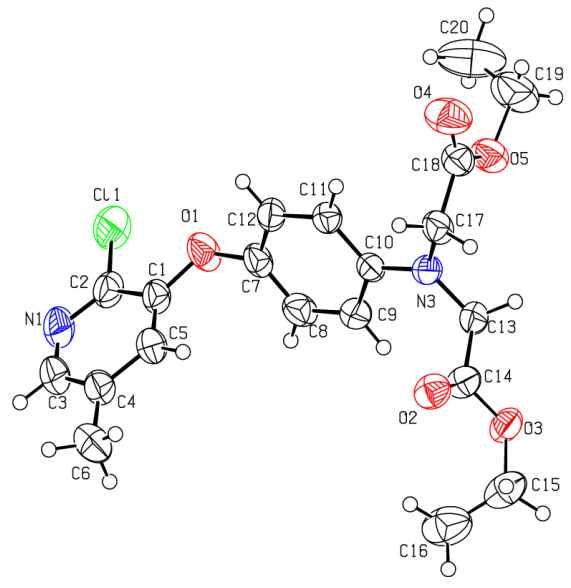

图 2 化合物 $\mathbf{F}$ 的分子结构

Figure 2 Molecular structure of compound $\mathbf{F}$

1.2 吡啶氧基苯胺基丙酸/乙酸乙酯对稗草和油菜除草 活性

采用稗草平皿法和油菜平皿法, 测定了目标化合物 对稗草和油菜根的除草活性, 化合物 B1 B18, C1 $\mathbf{C 3}, \mathbf{D}$ 和 $\mathbf{F}$ 的培养血除草活性数据见表 1 .

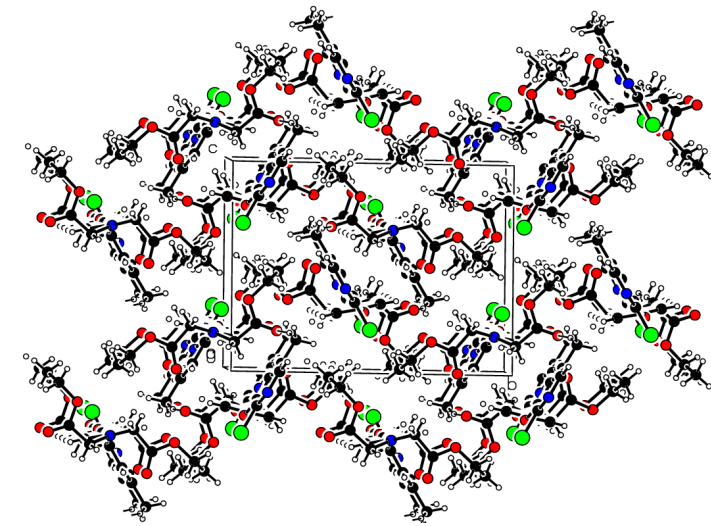

图 3 化合物 $\mathbf{F}$ 的晶狍堆积图

Figure 3 Part of the crystal packing in compound $\mathbf{F}$

从表 1 可知, 在培养皿生物测定中, 新化合物对稗 草表现出一定的除草活性，特别是化合物 B5、B12、

B14、B16 的 $\mathrm{IC}_{50}$ 都低于 $50 \mathrm{mg} / \mathrm{L}, \mathbf{B 5}$ 的 $\mathrm{IC}_{50}$ 为 27.692 $\mathrm{mg} / \mathrm{L}$, 对稗草茎的除草活性与对照药剂氟吡甲禾灵相 当. 初步的构效关系比较发现, 新化合物吡定环上引入 强吸电子取代基硝基时表现出更强的除草活性; 而且, 吡啶环上 3 位有强吸电子取代基的生物活性高于 5 位.

表 1 化合物 B1 $\sim$ B18, C1 $\sim \mathbf{C 3}, \mathrm{D}$ 和 F 的培养血除草活性

Table 1 Plate herbicidal activity of compounds B1 $\sim$ B18, C1 $\sim \mathbf{C 3}, \mathbf{D}$ and $\mathbf{F}$

\begin{tabular}{|c|c|c|c|c|c|c|c|}
\hline \multicolumn{2}{|c|}{ Compd } & \multicolumn{3}{|c|}{ 稗草 } & \multicolumn{3}{|c|}{ 油菜 } \\
\hline No. & $X$ & 回归曲线 & $r$ & $\mathrm{IC}_{50} /\left(\mathrm{mg} \cdot \mathrm{L}^{-1}\right)$ & 回归曲线 & $r$ & $\mathrm{IC}_{50} /\left(\mathrm{mg} \cdot \mathrm{L}^{-1}\right)$ \\
\hline B1 & $3-\mathrm{Cl}-5-\mathrm{CF}_{3}$ & $y=1.623 x+1.962$ & 0.981 & 74.480 & $y=1.951 x+1.656$ & 0.993 & 51.807 \\
\hline B2 & $5-\mathrm{CF}_{3}$ & $y=0.874 x+3.477$ & 0.995 & 55.304 & $y=2.484 x+0.369$ & 0.996 & 73.180 \\
\hline B3 & $3-\mathrm{CF}_{3}$ & $y=0.925 x+3.168$ & 0.998 & 103.39 & $y=2.167 x+1.253$ & 0.98 & 55.637 \\
\hline B4 & $3-\mathrm{Cl}$ & $y=1.316 x+2.067$ & 0.98 & 196.604 & $y=2.359 x+0.691$ & 0.986 & 67.120 \\
\hline B5 & $5-\mathrm{NO}_{2}$ & $y=1.070 x+3.457$ & 0.976 & 27.692 & $y=1.088 x+3.311$ & 0.991 & 35.727 \\
\hline B6 & $3,5-\mathrm{Br}_{2}$ & $y=2.066 x+1.507$ & 0.992 & 113.381 & $y=1.433 x+2.704$ & 0.992 & 39.979 \\
\hline B7 & $4-\mathrm{Br}$ & $y=2.475 x+0.562$ & 0.972 & 62.088 & $y=1.554 x+2.430$ & 0.988 & 45.132 \\
\hline B8 & $3-\mathrm{F}-5-\mathrm{CH}_{3}$ & $y=2.176 x+0.770$ & 0.995 & 88.005 & $y=1.985 x+1.585$ & 0.994 & 52.464 \\
\hline B9 & $5-\mathrm{COOCH}_{3}$ & $y=2.531 x+0.532$ & 0.992 & 58.289 & $y=1.842 x+2.049$ & 0.981 & 39.981 \\
\hline B10 & $5-\mathrm{Cl}$ & $y=0.922 x+3.274$ & 0.999 & 74.392 & $y=1.637 x+2.301$ & 0.967 & 44.540 \\
\hline B11 & $3-\mathrm{Br}-5-\mathrm{Cl}$ & $y=1.379 x+2.548$ & 0.983 & 59.917 & $y=1.628 x+2.321$ & 0.974 & 44.252 \\
\hline B12 & $3-\mathrm{NO}_{2}-5-\mathrm{Br}$ & $y=2.033 x+1.624$ & 0.975 & 45.777 & $y=1.280 x+3.003$ & 0.979 & 36.325 \\
\hline B13 & $6-\mathrm{F}$ & $y=1.523 x+1.196$ & 0.993 & 314.823 & $y=3.491 x-1.002$ & 0.98 & 55.441 \\
\hline B14 & $3-\mathrm{Cl}-5-\mathrm{NO}_{2}$ & $y=1.399 x+2.639$ & 0.998 & 48.646 & $y=2.942 x-0.742$ & 0.994 & 89.450 \\
\hline B15 & $3,5-\mathrm{Cl}_{2}$ & $y=3.236 x-0.926$ & 0.99 & 67.789 & $y=1.905 x+1.126$ & 0.996 & 108.202 \\
\hline B16 & $3-\mathrm{NO}_{2}$ & $y=2.210 x+1.359$ & 0.987 & 44.446 & $y=3.022 x-0.492$ & 0.993 & 65.613 \\
\hline B17 & $3-\mathrm{F}$ & $y=2.836 x-0.100$ & 0.994 & 62.793 & $y=1.922 x+1.767$ & 0.976 & 48.102 \\
\hline B18 & $3-\mathrm{Cl}-5-\mathrm{NH}_{2}$ & $y=1.880 x+1.293$ & 0.988 & 93.796 & $y=2.963 x-0.884$ & 0.999 & 96.782 \\
\hline C1 & - & - & - & $>500$ & $y=2.791 x-1.899$ & 0.981 & 296.25 \\
\hline $\mathrm{C2}$ & - & $y=4.299 x-5.513$ & 0.978 & 278.74 & - & - & $>500$ \\
\hline C3 & - & $y=3.607 x-4.415$ & 0.999 & 343.065 & $y=1.368 x+1.997$ & 0.964 & 156.86 \\
\hline D & - & $y=3.105 x-2.799$ & 0.995 & 325.025 & $y=3.970 x-4.763$ & 0.995 & 287.98 \\
\hline $\mathbf{F}$ & - & - & - & $>500$ & $y=2.089 x+0.860$ & 0.997 & 95.862 \\
\hline 氟吡甲禾灵 & - & $y=0.874 x+3.750$ & 0.993 & 26.959 & $y=1.272 x+2.126$ & 0.996 & 182.122 \\
\hline
\end{tabular}


值得一提的是, 合成的大部分化合物对油菜都有较 强的抑制作用, 优于氟吡甲禾灵. 从生物测定结果看, 吡啶环上取代基的差异与对油菜根的生物活性之间没 有明显的相关性.

本研究进一步测定了化合物 B5, B16, C1 和 $\mathbf{F}$ 的室 内盆栽除草活性. 结果表明化合物 B5, B16 表现出较好 的除草活性, 对稗草的除草活性略低于氟吡甲禾灵. 而 当丙酸乙酯被乙酸乙酯取代后, 除草活性显著下降. 测 定的 5 个化合物对油菜的盆栽除草活性都不高.

表 2 化合物 B5, B16, C1 和 F 的室内盆栽除草活性

Table 2 Greenhouse herbicidal activities of the compounds B5, B16, $\mathbf{C 1}$ and $\mathbf{F}$

\begin{tabular}{|c|c|c|c|}
\hline \multirow{2}{*}{ Compd. } & \multirow{2}{*}{ 剂量/ $\left(g \bullet h a^{-1}\right)$} & \multicolumn{2}{|c|}{ 抑制率/\% } \\
\hline & & 稗草 & 油菜 \\
\hline \multirow[t]{3}{*}{ B5 } & 400 & 88.43 & 57.66 \\
\hline & 200 & 72.54 & 39.01 \\
\hline & 100 & 54.17 & 17.78 \\
\hline \multirow[t]{3}{*}{ B16 } & 400 & 66.67 & 47.99 \\
\hline & 200 & 54.42 & 29.51 \\
\hline & 100 & 38.61 & 19.08 \\
\hline \multirow[t]{3}{*}{ 氟吡甲禾灵 } & 400 & 95.35 & 30.45 \\
\hline & 200 & 79.37 & 13.76 \\
\hline & 100 & 61.68 & 7.82 \\
\hline \multirow[t]{3}{*}{$\mathrm{C} 1$} & 400 & 24.09 & 16.02 \\
\hline & 200 & 20.88 & 4.81 \\
\hline & 100 & 15.83 & 1.11 \\
\hline \multirow[t]{3}{*}{ F } & 400 & 25.08 & 17.82 \\
\hline & 200 & 18.06 & 12.88 \\
\hline & 100 & 9.52 & 8.72 \\
\hline
\end{tabular}

\section{2 结论}

本文以 2-氯吡啶衍生物和对胺基苯酚等作为原料, 合成了 23 个全新吡啶氧基苯胺基丙酸/乙酸乙酯类化合 物, 产物经过核磁共振氢谱、高分辨质谱和 $\mathrm{X}$ 晶体衍射 确认了结构, 并采用培养血法和盆栽试验测定了新化合 物对稗草和油菜的室内生物活性. 结果表明大部分目标 化合物对稗草表现出中等到优秀的除草活性, 其中化合 物 B5 活性最高, 与氟吡甲禾灵的除草活性相当. 构效 关系比较发现, 吡啶环上有硝基取代基时表现出更强的 除草活性; 而且, 吡啶环上 3 位有强吸电子取代基的除 草活性高于 5 位. 这个结果提供了一个有用的信息, 即 在这类除草剂的芳环 3 位引入强吸电子基团可能会提高 除草活性. 化合物 B5 不论是在培养血还是在盆栽的试 验中, 对稗草都表现出与氟吡甲禾灵相当的除草活性, 所以, 化合物 B5 可以作为一个开发新除草剂的先导化 合物.

\section{3 实验部分}

\section{1 仪器与试剂}

申光熔点仪(上海精密科学仪器有限公司, 温度计 未校正); 高分辨质谱仪(Thermo Scientific 的 LTQ Orbitrap Discovery, Bremen, Germany); ${ }^{1} \mathrm{H}$ NMR(内标为 $\mathrm{TMS}$, 溶剂为 $\mathrm{CDCl}_{3}$ 和 DMSO- $d_{6}$ ) 使用 Bruker $\mathrm{AV} 600$ 和 Bruker AV 400 核磁共振仪测定; X 单晶衍射仪(Bruker SMART CCD-based diffractometer). 薄层和柱层析用硅 胶均为青岛海洋化工厂产品. 实验所用试剂均为国产分 析纯试剂.

\section{2 合成方法}

2-[4-(3-氯-5-三氟甲基-2-吡啶氧基)苯胺基]丙酸乙 酯(B1)参考文献[8]合成. 在带有氮气装置的 $100 \mathrm{~mL}$ 三 口瓶中, 加入 $40 \mathrm{~mL}$ DMF, 依次加入 $2.18 \mathrm{~g}$ 对胺基苯酚 和 $1.92 \mathrm{~g}$ 叔丁醇钠, 在室温下, 搅拌约 $2 \mathrm{~h}$, 再加入 4.32 $\mathrm{g} 2,3-\mathrm{Cl}_{2}-5-\mathrm{CF}_{3}$ 吡啶和 $2.78 \mathrm{~g} \mathrm{~K}_{2} \mathrm{CO}_{3}$, 继续摚拌反应. 用 $\mathrm{TLC}$ 检测反应，待反应结束后，减压蒸馏出 DMF，用 50 $\mathrm{mL}$ 氯仿溶解, 萃取, 取油层, 无水硫酸钠干燥, 静置过 夜, 过滤浓缩得黑褐色粉末. 即为 4-(3-氯-5-三氟甲基2-吡啶氧基)苯胺, 称重 $4.52 \mathrm{~g}$, 收率约为 78.4\%.

将上述 $4.52 \mathrm{~g}$ 4-(3-氯-5-三氟甲基-2-吡啶氧基)苯胺 和 $2.73 \mathrm{~g} \mathrm{2}$-氯丙酸乙酯置于 $100 \mathrm{~mL}$ 圆底烧瓶中, 加入 $2.76 \mathrm{~g} \mathrm{~K}_{2} \mathrm{CO}_{3}$ 和催化剂量的 $\mathrm{KI}$, 加入 $40 \mathrm{~mL} \mathrm{DMF}$ 作为溶 剂, 加热至 $100{ }^{\circ} \mathrm{C}$, 搅拌反应. 用薄层色谱(TLC)检测 反应. 待反应结束后, 减压蒸馏出 $N, N$-二甲基甲酰胺 (DMF), 用 $50 \mathrm{~mL}$ 氯仿溶解, 萃取, 取油层, 无水硫酸钠 干燥, 静置过夜, 过滤浓缩得到黏稠液体, 用乙酸乙酯: 石油醚进行柱层析分离, 得到红棕色固体, 即为 B1, 重 $4.27 \mathrm{~g}$, 收率 $84.8 \%$. 化合物 B2 $\sim$ B17 都按此方法合成.

化合物 B14 (1.6 mmol)溶解在 $60 \mathrm{~mL}$ 乙醇和 $12 \mathrm{~mL}$ 蒸馏水中, 投入氯化铵 $4.8 \mathrm{~g}$ 和还原铁粉 $3.25 \mathrm{~g}$. 加热回 流 $1 \mathrm{~h}$, 用薄层色谱(TLC)检测反应, 趁热过滤即得到纯 的 B18.

化合物 C1 $\sim \mathbf{C 3}$ 的合成采用 B1 的方法, 只是用 2氯乙酸乙酯替代 2 -氯丙酸乙酯.

中间体 $\mathbf{A}$ 与三倍量的 2-氯丙酸乙酯反应得到化合 物 D. 采用同样的方法，以 2-氯-3-氟-5-甲基吡啶为原料 合成得到化合物 $\mathbf{F}$.

\section{3 化合物的表征数据}

2-[4-(3-氯-5-三氟甲基-2-吡啶氧基)苯胺基]丙酸乙 酯(B1): 红棕色固体, 收率 $84.8 \%$. m.p. $62.4 \sim 64.2{ }^{\circ} \mathrm{C}$; ${ }^{1} \mathrm{H}$ NMR $\left(400 \mathrm{MHz}, \mathrm{CDCl}_{3}\right) \delta: 8.29(\mathrm{~s}, 1 \mathrm{H}), 7.97(\mathrm{~s}, 1 \mathrm{H})$, $7.01(\mathrm{~d}, J=8.0 \mathrm{~Hz}, 2 \mathrm{H}), 6.68(\mathrm{~d}, J=8.0 \mathrm{~Hz}, 2 \mathrm{H}), 4.25(\mathrm{~d}$, $J=7.2 \mathrm{~Hz}, 3 \mathrm{H}), 4.14$ (s, $1 \mathrm{H}), 1.52(\mathrm{~d}, J=6.4 \mathrm{~Hz}, 3 \mathrm{H}), 1.30$ 
(t, $J=6.4 \mathrm{~Hz}, 3 \mathrm{H}$ ). HRMS calcd for $\mathrm{C}_{17} \mathrm{H}_{17} \mathrm{ClF}_{3} \mathrm{~N}_{2} \mathrm{O}_{3}[\mathrm{M}+$

$\mathrm{H}]^{+}$389.0874, found 389.0876 .

2-[4-(5-三氟甲基-2-吡啶氧基)苯胺基]丙酸乙酯 (B2): 白色固体, 收率 67\%. m.p. 50.2 51.4 ${ }^{\circ} \mathrm{C} ;{ }^{1} \mathrm{H}$ NMR (400 MHz, DMSO- $d_{6}$ ) $\delta: 9.01(\mathrm{~s}, 1 \mathrm{H}), 8.63 \sim 8.48$ (m, 1H), $8.15(\mathrm{~s}, 1 \mathrm{H}), 7.10(\mathrm{t}, J=9.6 \mathrm{~Hz}, 1 \mathrm{H}), 6.93(\mathrm{t}, J=$ $9.2 \mathrm{~Hz}, 2 \mathrm{H}), 6.58(\mathrm{~s}, 2 \mathrm{H}), 6.09$ (dd, $J=9.6,4.0 \mathrm{~Hz}, 1 \mathrm{H})$, $4.11(\mathrm{~d}, J=6.8 \mathrm{~Hz}, 2 \mathrm{H}), 4.04$ (s, 1H), 1.39 (d, $J=6.4 \mathrm{~Hz}$, $3 \mathrm{H}), 1.18(\mathrm{t}, J=6.4 \mathrm{~Hz}, 3 \mathrm{H})$. HRMS calcd for $\mathrm{C}_{17} \mathrm{H}_{18} \mathrm{~F}_{3} \mathrm{~N}_{2} \mathrm{O}_{3}[\mathrm{M}+\mathrm{H}]^{+}$355.1264, found 355.1262.

2-[4-(3-三氟甲基-2-吡啶氧基)苯胺基]丙酸乙酯 (B3): 白色固体, 收率 88.8\%. m.p. $76 \sim 77{ }^{\circ} \mathrm{C} ;{ }^{1} \mathrm{H}$ NMR $\left(400 \mathrm{MHz}\right.$, DMSO- $\left.d_{6}\right) \delta: 8.34(\mathrm{~d}, J=4.4 \mathrm{~Hz}, 1 \mathrm{H}), 8.20(\mathrm{~d}$, $J=7.6 \mathrm{~Hz}, 1 \mathrm{H}), 7.28 \sim 7.23(\mathrm{~m}, 1 \mathrm{H}), 6.88(\mathrm{~d}, J=8.8 \mathrm{~Hz}$, $2 \mathrm{H}), 6.57(\mathrm{~d}, J=8.8 \mathrm{~Hz}, 2 \mathrm{H}), 6.02(\mathrm{~d}, J=8.4 \mathrm{~Hz}, 1 \mathrm{H}), 4.10$ (q, $J=7.2 \mathrm{~Hz}, 2 \mathrm{H}), 4.02(\mathrm{q}, J=7.2 \mathrm{~Hz}, 1 \mathrm{H}), 1.38(\mathrm{~d}, J=$ $6.8 \mathrm{~Hz}, 3 \mathrm{H}), 1.18(\mathrm{t}, J=7.2 \mathrm{~Hz}, 3 \mathrm{H})$. HRMS calcd for $\mathrm{C}_{17} \mathrm{H}_{18} \mathrm{~F}_{3} \mathrm{~N}_{2} \mathrm{O}_{3}[\mathrm{M}+\mathrm{H}]^{+}$355.1264, found 355.1268.

2-[4-(3-氯-2-吡定氧基)苯胺基]丙酸乙酯(B4): 白色 固体, 收率 80.9\%. m.p. $76 \sim 77.5{ }^{\circ} \mathrm{C} ;{ }^{1} \mathrm{H}$ NMR $(600$ $\left.\mathrm{MHz}, \mathrm{CDCl}_{3}\right) \delta: 8.02(\mathrm{dd}, J=4.8,1.8 \mathrm{~Hz}, 1 \mathrm{H}), 7.73(\mathrm{dd}$, $J=7.8,1.2 \mathrm{~Hz}, 1 \mathrm{H}), 7.02 \sim 6.98(\mathrm{~m}, 2 \mathrm{H}), 6.92(\mathrm{dd}, J=7.8$, $4.8 \mathrm{~Hz}, 1 \mathrm{H}), 6.69 \sim 6.63(\mathrm{~m}, 2 \mathrm{H}), 4.21(\mathrm{q}, J=7.2 \mathrm{~Hz}, 2 \mathrm{H})$, 4.11 (q, $J=6.6 \mathrm{~Hz}, 1 \mathrm{H}), 1.49$ (d, $J=6.6 \mathrm{~Hz}, 3 \mathrm{H}), 1.28$ (t, $J=7.2 \mathrm{~Hz}, 3 \mathrm{H})$. HRMS calcd for $\mathrm{C}_{16} \mathrm{H}_{18} \mathrm{ClN}_{2} \mathrm{O}_{3}[\mathrm{M}+\mathrm{H}]^{+}$ 321.1000 , found 321.1006 .

2-[4-(5-硝基-2-吡啶氧基)苯胺基]丙酸乙酯(B5): 浅 黄色固体, 收率 89.6\%. m.p. $64 \sim 66{ }^{\circ} \mathrm{C} ;{ }^{1} \mathrm{H}$ NMR (400 MHz, DMSO- $\left.d_{6}\right) \delta: 9.02(\mathrm{~d}, J=2.8 \mathrm{~Hz}, 1 \mathrm{H}), 8.57$ (dd, $J=$ 9.2, $2.8 \mathrm{~Hz}, 1 \mathrm{H}), 7.11(\mathrm{~d}, J=9.2 \mathrm{~Hz}, 1 \mathrm{H}), 6.94$ (d, $J=8.8$ $\mathrm{Hz}, 2 \mathrm{H}), 6.59$ (d, $J=8.8 \mathrm{~Hz}, 2 \mathrm{H}), 6.11(\mathrm{~d}, J=8.4 \mathrm{~Hz}, 1 \mathrm{H})$, $4.11(\mathrm{~d}, J=7.2 \mathrm{~Hz}, 2 \mathrm{H}), 4.06 \sim 4.01(\mathrm{~m}, 1 \mathrm{H}), 1.39$ (d, $J=$ $6.8 \mathrm{~Hz}, 3 \mathrm{H}), 1.19$ (d, $J=7.2 \mathrm{~Hz}, 3 \mathrm{H})$. HRMS calcd for $\mathrm{C}_{16} \mathrm{H}_{18} \mathrm{ClN}_{2} \mathrm{O}_{3}[\mathrm{M}+\mathrm{H}]^{+}$332.1241, found 332.1242.

2-[4-(3,5-二溴-2-吡啶氧基)苯胺基]丙酸乙酯(B6): 白色固体, 收率 88.4\%. m.p. $68 \sim 69{ }^{\circ} \mathrm{C} ;{ }^{1} \mathrm{H}$ NMR $(400$ $\left.\mathrm{MHz}, \mathrm{CDCl}_{3}\right) \delta: 8.09(\mathrm{~d}, J=2.4 \mathrm{~Hz}, 1 \mathrm{H}), 8.04(\mathrm{~d}, J=2.4$ $\mathrm{Hz}, 1 \mathrm{H}), 7.00 \sim 6.97(\mathrm{~m}, 2 \mathrm{H}), 6.69 \sim 6.67(\mathrm{~m}, 2 \mathrm{H}), 4.23(\mathrm{q}$, $J=7.2 \mathrm{~Hz}, 3 \mathrm{H}), 4.13(\mathrm{q}, J=6.8 \mathrm{~Hz}, 1 \mathrm{H}), 1.51$ (d, $J=6.8$ $\mathrm{Hz}, 3 \mathrm{H}), 1.29(\mathrm{t}, J=7.2 \mathrm{~Hz}, 3 \mathrm{H})$. HRMS calcd for $\mathrm{C}_{16} \mathrm{H}_{17} \mathrm{Br}_{2} \mathrm{~N}_{2} \mathrm{O}_{3}[\mathrm{M}+\mathrm{H}]^{+}$442.9600, found 442.9603.

2-[4-(4-溴-2-吡啶氧基)苯胺基]丙酸乙酯(B7): 浅黄 色液体, 收率 78.8\%. ${ }^{1} \mathrm{H}$ NMR (400 MHz, DMSO- $\left.d_{6}\right) \delta$ : $8.19(\mathrm{~d}, J=4.0 \mathrm{~Hz}, 1 \mathrm{H}), 6.91(\mathrm{~d}, J=8.0 \mathrm{~Hz}, 2 \mathrm{H})$, 6.75-6.77 (m, 2H), 6.66 (d, $J=4.0 \mathrm{~Hz}, 2 \mathrm{H}), 4.22$ (q, $J=$
$8.0 \mathrm{~Hz}, 2 \mathrm{H}), 4.12(\mathrm{q}, J=8.0 \mathrm{~Hz}, 1 \mathrm{H}), 1.51(\mathrm{~d}, J=8.0 \mathrm{~Hz}$, $3 \mathrm{H}), 1.28(\mathrm{t}, J=6.0 \mathrm{~Hz}, 3 \mathrm{H})$. HRMS calcd for $\mathrm{C}_{16} \mathrm{H}_{18}-$ $\mathrm{BrN}_{2} \mathrm{O}_{3}[\mathrm{M}+\mathrm{H}]^{+}$365.0495, found 365.0493.

2-[4-(3-氟-5-甲基-2-吡啶氧基)苯胺基]丙酸乙酯 (B8): 浅黄色液体, 收率 79.6\%. ${ }^{1} \mathrm{H}$ NMR $(400 \mathrm{MHz}$, DMSO- $\left.d_{6}\right) \delta: 7.90(\mathrm{~d}, J=2.4 \mathrm{~Hz}, 1 \mathrm{H}), 6.91 \sim 6.88(\mathrm{~m}, 3 \mathrm{H})$, $6.67(\mathrm{~d}, J=8.0 \mathrm{~Hz}, 2 \mathrm{H}), 4.21(\mathrm{q}, J=8.0 \mathrm{~Hz}, 2 \mathrm{H}), 4.11(\mathrm{q}$, $J=7.6 \mathrm{~Hz}, 1 \mathrm{H}), 2.23(\mathrm{~s}, 3 \mathrm{H}), 1.50(\mathrm{~d}, J=6.8 \mathrm{~Hz}, 3 \mathrm{H}), 1.27$ (t, $J=6.0 \mathrm{~Hz}, 3 \mathrm{H}$ ). HRMS calcd for $\mathrm{C}_{17} \mathrm{H}_{20} \mathrm{FN}_{2} \mathrm{O}_{3}[\mathrm{M}+$ $\mathrm{H}]^{+} 319.1452$, found 319.1455 .

2-[4-(5-甲酸甲酯-2-吡啶氧基)苯胺基]丙酸乙酯 (B9): 浅黄色液体, 收率 $85.8 \% .{ }^{1} \mathrm{H}$ NMR $(600 \mathrm{MHz}$, $\left.\mathrm{CDCl}_{3}\right) \delta: 8.83(\mathrm{dd}, J=2.4,1.2 \mathrm{~Hz}, 1 \mathrm{H}), 8.23(\mathrm{dd}, J=8.4$, $2.4 \mathrm{~Hz}, 1 \mathrm{H}), 7.03 \sim 6.96(\mathrm{~m}, 2 \mathrm{H}), 6.86(\mathrm{dd}, J=9.0,0.6 \mathrm{~Hz}$, $1 \mathrm{H}), 6.72 \sim 6.60(\mathrm{~m}, 2 \mathrm{H}), 4.22(\mathrm{q}, J=7.2 \mathrm{~Hz}, 3 \mathrm{H}), 4.12(\mathrm{q}$, $J=6.6 \mathrm{~Hz}, 1 \mathrm{H}), 3.92$ (s, $3 \mathrm{H}), 1.50$ (d, $J=7.2 \mathrm{~Hz}, 3 \mathrm{H}), 1.28$ (t, $J=7.2 \mathrm{~Hz}, 3 \mathrm{H}$ ). HRMS calcd for $\mathrm{C}_{18} \mathrm{H}_{21} \mathrm{~N}_{2} \mathrm{O}_{5}[\mathrm{M}+\mathrm{H}]^{+}$ 345.1445 , found 345.1443 .

2-[4-(5-氯-2-吡啶氧基)苯胺基]丙酸乙酯(B10): 白 色固体, 收率 79.4\%. m.p. $45.3 \sim 47.6{ }^{\circ} \mathrm{C} ;{ }^{1} \mathrm{H}$ NMR (600 $\left.\mathrm{MHz}, \mathrm{CDCl}_{3}\right) \delta: 8.13(\mathrm{dd}, J=2.4,0.6 \mathrm{~Hz}, 1 \mathrm{H}), 7.60$ (dd, $J=8.4,2.4 \mathrm{~Hz}, 1 \mathrm{H}), 7.02 \sim 6.92(\mathrm{~m}, 2 \mathrm{H}), 6.80$ (dd, $J=9.0$, $0.6 \mathrm{~Hz}, 1 \mathrm{H}), 6.69 \sim 6.60(\mathrm{~m}, 2 \mathrm{H}), 4.22(\mathrm{q}, J=7.2 \mathrm{~Hz}, 2 \mathrm{H})$, $4.18(\mathrm{~d}, J=6.0 \mathrm{~Hz}, 1 \mathrm{H}), 4.11$ (q, $J=6.6 \mathrm{~Hz}, 1 \mathrm{H}), 1.49$ (d, $J=6.6 \mathrm{~Hz}, 3 \mathrm{H}), 1.28$ (t, $J=7.2 \mathrm{~Hz}, 3 \mathrm{H})$. HRMS calcd for $\mathrm{C}_{16} \mathrm{H}_{18} \mathrm{ClN}_{2} \mathrm{O}_{3}[\mathrm{M}+\mathrm{H}]^{+}$321.1000, found 321.1000.

2-[4-(3-澳-5-氯-2-吡啶氧基)苯胺基]丙酸乙酯(B11): 白色固体, 收率 5.6\%. m.p. $68 \sim 69{ }^{\circ} \mathrm{C} ;{ }^{1} \mathrm{H}$ NMR $(600$ $\left.\mathrm{MHz}, \mathrm{CDCl}_{3}\right) \delta: 8.00(\mathrm{~d}, J=2.4 \mathrm{~Hz}, 1 \mathrm{H}), 7.91(\mathrm{~d}, J=2.4$ $\mathrm{Hz}, 1 \mathrm{H}), 7.02 \sim 6.95(\mathrm{~m}, 2 \mathrm{H}), 6.69 \sim 6.62(\mathrm{~m}, 2 \mathrm{H}), 4.22(\mathrm{q}$, $J=7.2 \mathrm{~Hz}, 3 \mathrm{H}), 4.11(\mathrm{q}, J=6.6 \mathrm{~Hz}, 1 \mathrm{H}), 1.49$ (d, $J=7.2$ $\mathrm{Hz}, 3 \mathrm{H}), 1.28$ (t, $J=7.2 \mathrm{~Hz}, 3 \mathrm{H})$. HRMS calcd for $\mathrm{C}_{16} \mathrm{H}_{17} \mathrm{BrClN}_{2} \mathrm{O}_{3}[\mathrm{M}+\mathrm{H}]^{+}$399.0106, found 399.0111.

2-[4-(3-硝基-5-澳-2-吡啶氧基)苯胺基]丙酸乙酯 (B12): 浅黄色液体, 收率 $87.6 \% .{ }^{1} \mathrm{H}$ NMR $(600 \mathrm{MHz}$, $\left.\mathrm{CDCl}_{3}\right) \delta: 8.45(\mathrm{~d}, J=2.4 \mathrm{~Hz}, 1 \mathrm{H}), 8.37(\mathrm{~d}, J=2.4 \mathrm{~Hz}$, $1 \mathrm{H}), 7.02 \sim 6.95(\mathrm{~m}, 2 \mathrm{H}), 6.69 \sim 6.61(\mathrm{~m}, 2 \mathrm{H}), 4.22(\mathrm{q}, J=$ $7.2 \mathrm{~Hz}, 2 \mathrm{H}), 4.12(\mathrm{t}, J=6.6 \mathrm{~Hz}, 1 \mathrm{H}), 1.50(\mathrm{~d}, J=6.6 \mathrm{~Hz}$, $3 \mathrm{H}), 1.28$ (t, $J=7.2 \mathrm{~Hz}, 3 \mathrm{H})$. HRMS calcd for $\mathrm{C}_{16} \mathrm{H}_{17} \mathrm{BrN}_{3} \mathrm{O}_{5}[\mathrm{M}+\mathrm{H}]^{+}$410.0346, found 410.0349.

2-[4-(6-氟-2-吡啶氧基)苯胺基]丙酸乙酯(B13): 浅 黄色液体，收率 $90.4 \% .{ }^{1} \mathrm{H}$ NMR $\left(600 \mathrm{MHz}, \mathrm{CDCl}_{3}\right) \delta$ : $7.55(\mathrm{dd}, J=7.8,1.2 \mathrm{~Hz}, 1 \mathrm{H}), 7.04 \sim 6.93(\mathrm{~m}, 3 \mathrm{H}), 6.66 \sim$ $6.59(\mathrm{~m}, 3 \mathrm{H}), 4.21(\mathrm{q}, J=7.2 \mathrm{~Hz}, 3 \mathrm{H}), 4.13 \sim 4.06(\mathrm{~m}$, $1 \mathrm{H}), 1.49$ (d, $J=6.6 \mathrm{~Hz}, 3 \mathrm{H}), 1.26(\mathrm{t}, J=7.2 \mathrm{~Hz}, 3 \mathrm{H})$. 
HRMS calcd for $\mathrm{C}_{16} \mathrm{H}_{18} \mathrm{FN}_{2} \mathrm{O}_{3}[\mathrm{M}+\mathrm{H}]^{+}$305.1296, found 305.1295 .

2-[4-(3-氯-5-硝基-2-吡啶氧基)苯胺基]丙酸乙酯 (B14): 浅黄色液体, 收率 86.4\%. ${ }^{1} \mathrm{H}$ NMR $(600 \mathrm{MHz}$, $\left.\mathrm{CDCl}_{3}\right) \delta: 8.89(\mathrm{~d}, J=2.4 \mathrm{~Hz}, 1 \mathrm{H}), 8.55(\mathrm{~d}, J=2.4 \mathrm{~Hz}$, $1 \mathrm{H}), 7.03 \sim 6.97(\mathrm{~m}, 2 \mathrm{H}), 6.71 \sim 6.63(\mathrm{~m}, 2 \mathrm{H}), 4.29(\mathrm{~d}, J=$ $7.8 \mathrm{~Hz}, 1 \mathrm{H}), 4.23$ (q, $J=7.2 \mathrm{~Hz}, 2 \mathrm{H}), 4.13$ (q, $J=7.2 \mathrm{~Hz}$, $1 \mathrm{H}), 1.50$ (d, $J=7.2 \mathrm{~Hz}, 3 \mathrm{H}), 1.29$ (t, $J=7.2 \mathrm{~Hz}, 3 \mathrm{H})$. HRMS calcd for $\mathrm{C}_{16} \mathrm{H}_{17} \mathrm{ClN}_{3} \mathrm{O}_{5}[\mathrm{M}+\mathrm{H}]^{+} 366.0851$, found 366.0852 .

2-[4-(3,5-二氯-2-吡啶氧基)苯胺基]丙酸乙酯(B15): 白色固体, 收率 84.2\%. m.p. 59.5 60.4 ${ }^{\circ} \mathrm{C} ;{ }^{1} \mathrm{H}$ NMR $\left(600 \mathrm{MHz}, \mathrm{CDCl}_{3}\right) \delta: 7.96(\mathrm{~d}, J=2.4 \mathrm{~Hz}, 1 \mathrm{H}), 7.74$ (d, $J=$ $2.4 \mathrm{~Hz}, 1 \mathrm{H}), 7.04 \sim 6.95(\mathrm{~m}, 2 \mathrm{H}), 6.70 \sim 6.58(\mathrm{~m}, 2 \mathrm{H}), 4.22$ (q, $J=7.2 \mathrm{~Hz}, 3 \mathrm{H}), 4.11(\mathrm{q}, J=7.2 \mathrm{~Hz}, 1 \mathrm{H}), 1.49$ (d, $J=$ $7.2 \mathrm{~Hz}, 3 \mathrm{H}), 1.28(\mathrm{t}, J=7.2 \mathrm{~Hz}, 3 \mathrm{H})$. HRMS calcd for $\mathrm{C}_{16} \mathrm{H}_{17} \mathrm{Cl}_{2} \mathrm{~N}_{2} \mathrm{O}_{3}[\mathrm{M}+\mathrm{H}]^{+}$355.0611, found 355.0620.

2-[4-(3-硝基-2-吡啶氧基)苯胺基]丙酸乙酯(B16): 浅黄色液体, 收率 $82.1 \% .{ }^{1} \mathrm{H}$ NMR $\left(600 \mathrm{MHz} \mathrm{CDCl}_{3}\right) \delta$ : $10.00(\mathrm{~s}, 1 \mathrm{H}), 8.51$ (dd, $J=8.4,1.8 \mathrm{~Hz}, 1 \mathrm{H}), 8.45$ (dd, $J=$ $4.8,1.8 \mathrm{~Hz}, 1 \mathrm{H}), 7.54 \sim 7.49(\mathrm{~m}, 2 \mathrm{H}), 6.95 \sim 6.90(\mathrm{~m}, 2 \mathrm{H})$, $6.80(\mathrm{dd}, J=8.4,4.2 \mathrm{~Hz}, 1 \mathrm{H}), 4.75$ (q, $J=6.6 \mathrm{~Hz}, 1 \mathrm{H})$, 4.24 (q, $J=7.2 \mathrm{~Hz}, 2 \mathrm{H}), 1.64$ (d, $J=7.2 \mathrm{~Hz}, 3 \mathrm{H}), 1.28$ (t, $J=7.2 \mathrm{~Hz}, 3 \mathrm{H})$. HRMS calcd for $\mathrm{C}_{16} \mathrm{H}_{18} \mathrm{~N}_{3} \mathrm{O}_{5}[\mathrm{M}+\mathrm{H}]^{+}$ 332.1241 , found 332.1247 .

2-[4-(3-氟-2-吡啶氧基)苯胺基]丙酸乙酯(B17): 黄 色固体, 收率 86.4\%. m.p. 92 93 ${ }^{\circ} \mathrm{C} ;{ }^{1} \mathrm{H}$ NMR (600 $\left.\mathrm{MHz} \mathrm{CDCl}_{3}\right) \delta: 8.06(\mathrm{dd}, J=4.2,1.8 \mathrm{~Hz}, 1 \mathrm{H}), 7.14 \sim 7.07$ $(\mathrm{m}, 2 \mathrm{H}), 6.92 \sim 6.87(\mathrm{~m}, 2 \mathrm{H}), 6.65 \sim 6.60(\mathrm{~m}, 2 \mathrm{H}), 4.21(\mathrm{q}$, $J=7.2 \mathrm{~Hz}, 3 \mathrm{H}), 4.13 \sim 4.07(\mathrm{~m}, 1 \mathrm{H}), 1.49(\mathrm{~d}, J=6.6 \mathrm{~Hz}$, $3 \mathrm{H}), \quad 1.27(\mathrm{t}, J=7.2 \mathrm{~Hz}, 3 \mathrm{H})$. HRMS calcd for $\mathrm{C}_{16} \mathrm{H}_{18} \mathrm{FN}_{2} \mathrm{O}_{3}[\mathrm{M}+\mathrm{H}]^{+}$305.1296, found 305.1295.

2-[4-(3-氯-5-氨基-2-吡啶氧基)苯胺基]丙酸乙酯 (B18): 浅黄色液体, 收率 78.9\%. ${ }^{1} \mathrm{H}$ NMR (600 MHz, $\left.\mathrm{CDCl}_{3}\right) \delta: 7.53(\mathrm{~d}, J=2.4 \mathrm{~Hz}, 1 \mathrm{H}), 7.15(\mathrm{~d}, J=2.4 \mathrm{~Hz}$, $1 \mathrm{H}), 6.97 \sim 6.91(\mathrm{~m}, 2 \mathrm{H}), 6.64 \sim 6.59(\mathrm{~m}, 2 \mathrm{H}), 4.20(\mathrm{q}, J=$ $7.2 \mathrm{~Hz}, 2 \mathrm{H}), 4.13 \sim 4.06(\mathrm{~m}, 2 \mathrm{H}), 3.53(\mathrm{~s}, 2 \mathrm{H}), 1.47$ (d, $J=$ $6.6 \mathrm{~Hz}, 3 \mathrm{H}), 1.27(\mathrm{t}, J=7.2 \mathrm{~Hz}, 3 \mathrm{H})$. HRMS calcd for $\mathrm{C}_{16} \mathrm{H}_{19} \mathrm{ClN}_{2} \mathrm{O}_{5}[\mathrm{M}+\mathrm{H}]^{+}$336.1109, found 336.1107.

2-[4-(5-硝基-2-吡啶氧基)苯胺基]乙酸乙酯(C1): 黄 色固体, 收率 81.3\%. m.p. 102 103 ${ }^{\circ} \mathrm{C} ;{ }^{1} \mathrm{H}$ NMR $(600$ $\left.\mathrm{MHz}, \mathrm{CDCl}_{3}\right) \delta: 9.05(\mathrm{~d}, J=3.0 \mathrm{~Hz}, 1 \mathrm{H}), 8.44$ (dd, $J=9.0$, $3.0 \mathrm{~Hz}, 1 \mathrm{H}), 7.01 \sim 6.96(\mathrm{~m}, 3 \mathrm{H}), 6.67 \sim 6.65(\mathrm{~m}, 2 \mathrm{H}), 4.40$ (t, $J=4.8 \mathrm{~Hz}, 1 \mathrm{H}), 4.27$ (q, $J=7.2 \mathrm{~Hz}, 2 \mathrm{H}), 3.92$ (d, $J=4.8$ $\mathrm{Hz}, 2 \mathrm{H}), 1.32$ (t, $J=7.2 \mathrm{~Hz}, 3 \mathrm{H})$. HRMS calcd for
$\mathrm{C}_{15} \mathrm{H}_{16} \mathrm{~N}_{3} \mathrm{O}_{5}[\mathrm{M}+\mathrm{H}]^{+}$318.1085, found 318.1087.

2-[4-(3-硝基-2-吡啶氧基)苯胺基]乙酸乙酯(C2): 红 色固体, 收率 79.5\%. m.p. 89 90 ${ }^{\circ} \mathrm{C} ;{ }^{1} \mathrm{H}$ NMR $(600$ $\left.\mathrm{MHz}, \mathrm{CDCl}_{3}\right) \delta: 10.00(\mathrm{~s}, 1 \mathrm{H}), 8.52$ (dd, $J=8.4,1.8 \mathrm{~Hz}$, $1 \mathrm{H}), 8.45(\mathrm{dd}, J=4.2,1.8 \mathrm{~Hz}, 1 \mathrm{H}), 7.53(\mathrm{~d}, J=9.0 \mathrm{~Hz}$, $2 \mathrm{H}), 6.99 \sim 6.94(\mathrm{~m}, 2 \mathrm{H}), 6.80(\mathrm{dd}, J=8.4,4.8 \mathrm{~Hz}, 1 \mathrm{H})$, 4.64 (s, 2H), 4.30 (q, $J=7.2 \mathrm{~Hz}, 2 \mathrm{H}), 1.32$ (t, $J=7.2 \mathrm{~Hz}$, $3 \mathrm{H})$. HRMS calcd for $\mathrm{C}_{15} \mathrm{H}_{16} \mathrm{~N}_{3} \mathrm{O}_{5}[\mathrm{M}+\mathrm{H}]^{+}$318.1085, found 318.1081 .

2-[4-(4-溴-2-吡啶氧基)苯胺基]乙酸乙酯 $(\mathbf{C 3})$ : 白色 固体, 收率 89.6\%. m.p. 70 71 ${ }^{\circ} \mathrm{C} ;{ }^{1} \mathrm{H}$ NMR (600 MHz, $\left.\mathrm{CDCl}_{3}\right) \delta: 8.19(\mathrm{~d}, J=6.0 \mathrm{~Hz}, 1 \mathrm{H}), 6.94 \sim 6.93(\mathrm{~m}, 2 \mathrm{H})$, $6.78 \sim 6.75(\mathrm{~m}, 2 \mathrm{H}), 6.66 \sim 6.64(\mathrm{~m}, 2 \mathrm{H}), 4.40(\mathrm{~s}, 1 \mathrm{H})$, 4.28 (q, $J=7.2 \mathrm{~Hz}, 2 \mathrm{H}), 3.92$ (d, $J=3.6 \mathrm{~Hz}, 2 \mathrm{H}), 1.32$ (t, $J=7.2 \mathrm{~Hz}, 3 \mathrm{H})$. HRMS calcd for $\mathrm{C}_{15} \mathrm{H}_{16} \mathrm{BrN}_{2} \mathrm{O}_{3}[\mathrm{M}+\mathrm{H}]^{+}$ 351.0339 , found 351.0339 .

2,2'-[4-(3-硝基-2-吡啶氧基)苯胺基]二乙酸二乙酯 (D): 黄色固体, 收率 80.6\%. m.p. 85 86 ${ }^{\circ} \mathrm{C} ;{ }^{1} \mathrm{H}$ NMR $\left(600 \mathrm{MHz}, \mathrm{CDCl}_{3}\right) \delta: 8.34 \sim 8.29(\mathrm{~m}, 2 \mathrm{H}), 7.10$ (dd, $J=$ $7.8,4.8 \mathrm{~Hz}, 1 \mathrm{H}), 7.05(\mathrm{~d}, J=9.0 \mathrm{~Hz}, 2 \mathrm{H}), 6.66$ (d, $J=9.6$ $\mathrm{Hz}, 2 \mathrm{H}), 4.22$ (q, $J=7.2 \mathrm{~Hz}, 4 \mathrm{H}), 4.15$ (s, 4H), 1.29 (t, $J=$ $7.2 \mathrm{~Hz}, 6 \mathrm{H})$. HRMS calcd for $\mathrm{C}_{19} \mathrm{H}_{22} \mathrm{~N}_{3} \mathrm{O}_{7}[\mathrm{M}+\mathrm{H}]^{+}$ 404.1453, found 404.1457.

2,2'-[4-(2-氯-5-甲基-3-吡啶氧基)苯胺基]二乙酸二 乙酯 $(\mathbf{F})$ : 浅黄色固体, 收率 70.4\%. m.p. 120 $121{ }^{\circ} \mathrm{C}$; ${ }^{1} \mathrm{H}$ NMR (400 MHz, $\left.\mathrm{CDCl}_{3}\right) \delta: 7.89(\mathrm{~s}, 1 \mathrm{H}), 6.91 \sim 6.94$ (m, 3H), 6.63 (d, $J=8.8 \mathrm{~Hz}, 2 \mathrm{H}), 4.23$ (q, $J=7.2 \mathrm{~Hz}, 4 \mathrm{H})$, 4.14 (s, 4H), 2.23 (s, 3H), 1.29 (t, $J=7.2 \mathrm{~Hz}, 6 \mathrm{H})$. HRMS calcd for $\mathrm{C}_{20} \mathrm{H}_{24} \mathrm{ClN}_{2} \mathrm{O}_{5}[\mathrm{M}+\mathrm{H}]^{+}$407.1368, found 407.1365.

\section{4 化合物 $F$ 的晶体衍射}

将 $45 \mathrm{mg}$ 化合物 $\mathbf{F}$ 置于 $20 \mathrm{~mL}$ 小瓶中, 分别用乙酸 乙酯/石油醚 $(V: V=1: 6)$ 将其溶解, 滤膜过滤, 用 Parafilm 膜将瓶口封好, 用针扎 5 个孔, 静置于阴凉处, 让溶剂缓慢挥发, 大约 $5 \mathrm{~d}$ 长出符合要求的晶体. 化合 物 $\mathbf{F}$ 的晶体结构数据已存于剑桥晶体结构数据库中, CCDC 号为 1501969 .

\section{5 室内除草活性的测定}

采用培养血法对新化合物进行室内除草活性测试, 方法参照文献[16２3]略有改进.

稗草和油菜的催芽处理: 先将稗草或油菜种子置于 蒸馏水浸泡 $24 \mathrm{~h}$, 然后置于垫有潮湿纱布的磁盘上, 转 移到 $30{ }^{\circ} \mathrm{C}$ 的光照培养箱中, 催芽 $24 \mathrm{~h}$. 从催好芽的种 子中, 选取大小均一并且饱满露白种子, 每个培养血 (直径 $7 \mathrm{~cm}$ ) 内置两张滤纸并放置 15 粒种子, 每一种药剂 
设置 6 个浓度, 三个重复, 然后向每个培养血加入 $4 \mathrm{~mL}$ 的药剂. 处理完毕后, 放在 $30{ }^{\circ} \mathrm{C}$ 的光照培养箱进行培 养, 约 $7 \mathrm{~d}$ 后, 稗草测量茎长, 油菜测量根长. 同时选用 氟吡甲禾灵作为对照药剂, 以清水加少量丙酮作为空白 对照.

化合物 B5, B16, C1 和 F 室内盆栽生物测定方法参 考文献[24 28]. 将萌发的稗草和油菜种子置于有土壤 的塑料碗中, 在室温下进行培养. 将化合物 B5, B16, C1 和 $\mathbf{F}$ 溶于 $1 \mathrm{~mL}$ 丙酮并滴加 1 滴吐温 80 , 然后加水配制 成乳浊液, 每个药剂设置 3 个浓度, 分别为 400, 200 和 $100 \mathrm{~g} / \mathrm{ha}$. 以 $300 \mathrm{~L} / \mathrm{ha}$ 的用水量对供试植物进行茎叶喷 雾, 药剂在植物第一片真叶展开后进行处理, 每个处理 重复 3 次. 药剂处理后 $10 \mathrm{~d}$ 称量植物地上部分鲜重, 计 算化合物对杂草的毒力.

辅助材料(Supporting Information) 目标化合物的核 磁共振图谱和化合物 $\mathrm{F}$ 的分子结构单晶衍射图及其结 构信息. 这些材料可以免费从本刊网站(http://siocjournal.cn/)上下载.

\section{References}

[1] Burton, J. D.; Gronwald, J. W.; Somers, D. A.; Connelly, J. A.; Gengenbach, B. G.; Wyse, D. L. Biochem. Biophys. Res. Commun. 1987, 148, 1039.

[2] Lichthenthaler, K.; Kobek, H. K.; Ishii, K. Z. Naturforsch. 1987, $42 c, 1275$.

[3] Delye, C.; Zhang, X. Q.; Michel, S.; Matejicek, A.; Powles, S. B. Plant Physiol. 2005, 137, 794.

[4] Gronward, J. W. Weed Sci. 1991, 39, 435.

[5] Singh, G.; Singh, M.; Singh, V. P. Indian J. Weed Sci. 2002, 34, 165.

[6] Tietze, L. F. Chem. Rev. 1996, 96, 115.

[7] Balme, G.; Bossharth, E.; Monteiro, N. Eur. J. Org. Chem. 2003, 21,4101 .

[8] Plowman, R. E.; Stonebridge, W. C.; Hawtree, J. N. Proceedings 1980 British Crop Protection Conference-Weeds, Brighton, England, 1980, p. 29.

[9] Menendez, J. C. Synthesis 2006, 2624.

[10] Ray, P. G.; Pews, R. G.; Flake, J. Proceedings 14th Conference Asian-Pacific Weed Science Society, Brisbane, Australia, 1993, p. 41.
[11] Dae, W. K.; Hae S. C.; Young, K. K.; Jae, W. R.; Jae, C. W.; Dong, W. K.; Jin, S. K. US 20030096706, 2003 [Chem. Abstr. 2003, 138, 364189].

[12] Coret, J. M. US 20050282706, 2005 [Chem. Abstr. 2005, 144, 33127].

[13] Graham, J. B.; Lindsay, E. C.; Graeme, J. F.; Wendy, A. J.; Jack, L.; Alexander, S.; Richard, B. W.; Keith, G. W. Bioorg. Med. Chem. Lett. 1997, 12, 1489

[14] Liu, H.; Wang, H. Q.; Liu, Z. J. Bioorg. Med. Chem. Lett. 2007, 17, 2203.

[15] Huang, T. H.; Tu, H. Y.; Zumureti, A.; Hou, C. J.; Zhang, A. D. ARKIVOR 2011, 2, 1

[16] Zhu, Y.-Q.; Zou, X.-M.; Hu, F.-Z.; Yao, C.-S.; Liu, B.; Li, Y.-H.; Yang, H.-Z. J. Agric. Food Chem. 2005, 53, 9566.

[17] Zhu, Y. Q.; Wu, C.; Li, H. B.; Zou, X. M.; Si, X. K.; Hu, F. Z.; Yang, H. Z. J. Agric. Food Chem. 2007, 55, 1364.

[18] Han, J. T.; Dong, H. B.; Xu, Z. H.; Lei, J. P.; Wang, M. A. Int. J. Mol. Sci. 2013, 14, 12484.

[19] Han, J. T.; Wang, J. M.; Dong, H. B.; Xu, Z. H.; Liu, B.; Wang, M. A. Chin. J. Org. Chem. 2013, 33, 596 (in Chinese). (韩金涛，王进敏，董宏波，徐志红，刘斌，王明安，有机化学， 2013, 33, 596.)

[20] Han, J. T.; Dong, H. B.; Wang, J. M.; Lei, J. P.; Wang, M. A.; Fang, J. X. Molecules 2011, 16, 2833

[21] Xu, Z. H.; Wang, J. M.; Han, J. T.; Liu, B.; Wang, M. A. Chin. J. Org. Chem. 2012, 32, 2134 (in Chinese). (徐志红，王进敏，韩金涛，刘斌，王明安，有机化学，2012，32， 2134.)

[22] Xu, Z. H.; Dong, H. B.; Liu, B.; Kong, L. Q.; Wang, M. A. Chin. J. Org. Chem. 2015, 35, 411 (in Chinese).

(徐志红，董宏波，刘斌，孔令青，王明安，有机化学，2015，35， 411.)

[23] Liu, B.; Dong, H. B.; Han, J. T.; Xu, Z. H.; Jin, S. H.; Wang, M. A. Chin. J. Org. Chem. 2013, 33, 2538 (in Chinese)

(刘斌，董宏波，韩金涛，徐志红，金淑惠，王明安，有机化学， 2013, 33, 2538.)

[24] Kluth, J.; Santel, H. J.; Schmidt, R. R. DE 3638151, 1987 [Chem. Abstr. 1988, 108, 111985].

[25] Wang, W.; He, H. W.; Zuo, N.; He, H. F.; Peng, H.; Tan, X. S. J. Agric. Food Chem. 2012, 60, 7581.

[26] He, H. W.; Chen, T.; Li, Y. J. J. Pestic. Sci. 2007, 32, 42.

[27] Peng, H.; Wang, T.; Xie, P.; Chen, T.; He, H. W.; Wan, J. J. Agric. Food Chem. 2007, 55, 1871.

[28] He, H. W.; Yuan, J. L.; Peng, H.; Chen, T.; Shen, P.; Wan, S. Q.; Li, Y. J.; Tan, H. L.; He, Y. H.; He, J. B.; Li, Y. J. Agric. Food Chem. 2011, 59, 4801. 